\title{
Elastic Registration of Biological Images Using Vector-Spline Regularization
}

\author{
Carlos Ó. S. Sorzano*, Philippe Thévenaz, and Michael Unser, Fellow, IEEE
}

\begin{abstract}
We present an elastic registration algorithm for the alignment of biological images. Our method combines and extends some of the best techniques available in the context of medical imaging. We express the deformation field as a B-spline model, which allows us to deal with a rich variety of deformations. We solve the registration problem by minimizing a pixelwise mean-square distance measure between the target image and the warped source. The problem is further constrained by way of a vector-spline regularization which provides some control over two independent quantities that are intrinsic to the deformation: its divergence, and its curl. Our algorithm is also able to handle soft landmark constraints, which is particularly useful when parts of the images contain very little information or when its repartition is uneven. We provide an optimal analytical solution in the case when only landmarks and smoothness considerations are taken into account. We have applied our approach to perform the elastic registration of images such as electrophoretic gels and fly embryos. The validation of the results by experts has been favorable in all cases.
\end{abstract}

\section{INTRODUCTION}

$\mathbf{P}$ UTTING two images into registration can be restated as finding a function (also called a deformation field) that performs the backward mapping of a target image onto a source image [1], [2]. This is a problem common to several molecular-biology disciplines like the analysis of microarrays [3], the analysis of genetic expression patterns [4], the recognition of protein folds [5], or the study of two-dimensional (2-D) electrophoretic gels [6].

The registration of biological images poses some challenging obstacles that need to be overcome. In most cases, the deformation is difficult to determine because it differs markedly from a simple rigid-body or affine transformation. Instead, biological images usually require local and nonlinear deformations, which only an elastic-deformation framework can handle properly. As additional difficulty, the information content of the images is sometimes distributed in uneven fashion (e.g., when there is no relevant information outside restricted image patches). At other times, it may be inherently impossible to register the two images since it is their difference that carries the useful information.

\footnotetext{
Manuscript received April 1, 2004; revised September 12, 2004. This work was supported in part by the "Universidad San Pablo-CEU" under Project 17/02 and in part by the Swiss National Foundation under Project 2153-06 693801. Asterisk indicates corresponding author.

*C. Ó. S. Sorzano is with the Biomedical Imaging Group, Swiss Federal Institute of Technology Lausanne (EPFL), Switzerland, and also with the Universidad San Pablo-CEU, Campus Urb. Montepríncipe s/n, E-28668 Boadilla del Monte, Madrid, Spain (e-mail: cos@cnb.uam.es).

P. Thévenaz and M. Unser are with the Biomedical Imaging Group, Swiss Federal Institute of Technology Lausanne (EPFL), Switzerland.

Digital Object Identifier 10.1109/TBME.2005.844030
}

Solutions have been proposed, mostly in the domain of medical image registration. Some a priori information about the deformation family is sometimes available (e.g., the deformation is smooth, or the deformation shows some kind of symmetry, or the deformation pattern is known). In addition, the use of landmarks is a common cure to the uneven distribution of information and to its potential inherent mismatch. Several algorithms deal exclusively with landmarks [7]-[11], while other algorithms ignore them; only a few authors propose a solution where the registration is guided by some combination of image and landmark contributions [12]. These facts motivated us to apply to biological image registration a generalization of some of the most recent works in medical image registration [12]-[14].

We select B-splines to model the images, which ensures their high-quality interpolation [15]. Similarly, we also use B-splines to model the deformation field [16]. In this way, a nonlinear elastic-deformation model can be produced while keeping a tight control on its level of detail. This deformation field is estimated through a minimization problem that includes three terms: the energy of the error between both images (data term), the error in the mapping of corresponding landmarks, and a regularization term that promotes a smooth deformation. We suggest to use masks to control the validity of the data term, to use soft landmarks to enforce a priori knowledge (possibly with some leeway), and to benefit from regularization, at least for those parts of the images where the absence of data and landmark information would else lead to undetermined registration.

We propose several new combinations of features: We suggest to assist the efficient optimization method of Levenberg and Marquardt by the accurate Broyden-Fletcher-Goldfarb-Shanno (BFGS) estimate of the Hessian; we also propose a dissimilarity measure that consists of no less than three terms. In addition, we develop a new analytical solution to the landmark-only problem when the deformation is B-spline-based and when the regularization term is truly vectorial.

After a discussion of related work in Section II, we introduce in Section III-A our measure of dissimilarity between images and take explicitly into account a priori information such as masks. We present our continuous B-spline-based representation of images and of the deformation in Sections III-B and III-C, respectively. We define the soft landmark constraints in Section III-D. We introduce a priori knowledge about the smoothness of the deformation via regularization in Section III-E. The analytical solution of the regularized registration problem in the absence of the image term is given in Section III-F. We present our multiresolution strategy in Section III-G. Our optimizer is inspired by a Levenberg-Marquardt nonlinear regression and 
is described in Section III-H. We develop in Section III-I the explicit computation of the derivatives needed by the optimizer. Finally, we present in Section IV experiments aimed at demonstrating the usefulness of our approach. We discuss the results of those experiments in Section V.

\section{STATE OF THE ART}

In this section, we briefly review previous registration methods. We identify the most important computational components, and justify the choices we have made for our implementation.

B-splines are piecewise-polynomial bases with a low computational cost, which makes them very attractive [17]. Because their approximation properties are optimal in a mathematically well-defined sense [18], [19], they are extremely useful to model many functions; in particular, the deformation field can be viewed as a set of several functions (one per coordinate) which in turn are modeled by a linear sum of weighted and shifted B-splines. The set of weights, which are called the B-spline coefficients, fully characterize the transformation.

A deformation model based on B-splines is very versatile and can generate a large variety of nonlinear elastic deformations, while remaining easy to handle [12]-[16], [20]. In this paper, we use B-splines to model the deformation field. In this way, a nonlinear elastic-deformation model can be produced while keeping a tight control on its level of detail. This is in contrast with nonparametric methods which do not seek to describe the deformation field by a controlled set of coefficients, but rather by partial differential equations [21], [22].

The role of landmarks is to anchor the deformation at some specific locations. A number of landmark-only registration algorithms have been developed because landmarks are essential in applications where images show very little information, or where the information in the target image is not the same as in the source images [8]-[10], [23]. Nevertheless, the accuracy of the landmarks is never subpixel when they are placed interactively, and suffers from other sources of noise when they result from an automatic selection procedure. To achieve high-accuracy registration, it is therefore crucial to be able to cope with inexact landmarks. We deal with soft landmarks by compounding their contribution with that of data and regularization terms.

In the restricted context of landmark-only registration, the deformation is known with perfect accuracy for corresponding landmarks, but is undetermined otherwise. Regularization is then called for to build a deformation field that is well-defined everywhere. The most classical regularization involves a term that is equivalent to the Laplacian of the deformation and that favors smoothness [24]-[27]. This regularization term is related to the mechanical stress of a stretched elastic material; the use of thin-plate splines for the interpolation of landmarks is also equivalent to the Laplacian [8], [9], [28]. Unfortunately, this standard regularization term considers only second-order derivatives and is blind to the "cross-talk" between components. As has been shown in [29], minimizing the energy of the Laplacian-equivalent is not sufficient to ensure maximal smoothness. The solution to the problem of fully exploiting the multidimensional nature of spline-based interpolation of vector fields, first proposed in [29], is to consider a combination of two regularization sub-terms, one based on the gradient of the divergence of the field, and another based on the gradient of its curl. The resulting vectorial regularization is a generalization of the standard one, which can still be obtained by a specific combination of divergence and curl. We show in this paper how to benefit from the extra freedom that results from choosing yet other combinations.

This vectorial regularization term has already been introduced in landmark-only image registration [10], [23], [30]. In the present paper, we propose two extensions. In the landmark-only case, we develop a new analytical solution to the problem of determining a B-spline-based deformation field that complies with vectorial regularization. Alternatively, we propose to incorporate three competing terms into the dissimilarity measure: landmark, regularization, and data terms. The combination of landmarks with regularization but without data is classical; the combination of landmarks with data but without regularization is encountered less often [12], as is the combination of data with regularization but without landmarks [24]. To the best of our knowledge, the simultaneous use of all three terms is performed in [31]-[33].

When the data term is taken into account, numeric optimization is required to minimize a measure of dissimilarity of the images. We have selected the energy of their difference as registration criterion, for which efficient optimization methods such as Levenberg-Marquardt are known to exist [34]. This optimizer adaptively adjusts the tradeoff between gradient-descent steps and quasi-Newton steps, which requires the estimate of the Hessian of the measure of dissimilarity with respect to the transformation parameters. We propose an approach whereby we apply the BFGS method to produce the estimate of the Hessian [35]. BFGS alone has already been used for image registration [13], [14], but has as yet never been combined with the Levenberg-Marquardt optimizer in this context.

The presence of the data term requires an interpolation model, not only to produce the consistent derivatives needed by the optimizer, but also to resample the image. Since high quality comes at a cost, practitioners have often compromised it by selecting linear interpolation. An even worse quality (essentially equivalent to that of nearest-neighbor) is observed for the partial-volume approach found in methods based on mutual information [36]. Here instead, we adopt the high-quality cubic B-spline interpolation scheme that was shown to be very successful for registration in [34].

Another advantage of B-splines is their suitability for building multiresolution algorithms thanks to the the two-scale property [37]. Coarser representations of the deformation field are computed using coarser representations of the images. Then, resolution increments are alternatively taken in the image space as well as in the deformation space, until a desired resolution is achieved in both. This multiresolution approach greatly increases the robustness and the speed of the algorithms, particularly in the context of gradient-based optimizers.

\section{Proposed Methodology}

Our registration methodology is based on the minimization of an energy functional that incorporates three terms: the measure of dissimilarity $E_{\text {img }}$ between the target image and the warped source image, the soft landmark constraints $E_{\mu}$, and a priori 
knowledge about the deformation field through the two independent measures $E_{\text {div }}$ and $E_{\text {rot }}$ that are related to the gradients of divergence and curl of the deformation field. Thus, the energy to minimize is a linear combination of these energy terms, as in

$$
E=w_{i} E_{\mathrm{img}}+w_{\mu} E_{\mu}+\left(w_{d} E_{\mathrm{div}}+w_{r} E_{\mathrm{rot}}\right) .
$$

\section{A. Data Term}

The goal of image registration is to find a function $\mathbf{g}(\mathbf{x})$ : $\mathbb{R}^{2} \rightarrow \mathbb{R}^{2}$ which maps coordinates from the target image $I_{t}$ onto the source image $I_{s}$, so that $I_{s}(\mathrm{~g}(\mathbf{x}))$ (a warped version of the source image) resembles $I_{t}(\mathbf{x})$ as much as possible. Several of the biological images that we deal with in this paper are nearly binary (for instance, 2-D electrophoretic gels) and are not appropriate for the use of histogram-based distance measures. In this case, we prefer to measure the energy of the difference between the target image and the warped image as

$$
E_{\text {img }}=\int_{\mathbf{x} \in \mathbb{R}^{2}}\left(I_{t}(\mathbf{x})-I_{s}(\mathbf{g}(\mathbf{x}))\right)^{2} \mathrm{~d} x \mathrm{~d} y .
$$

From a practical point of view, it is more convenient to approximate the integral in (2) by a discrete sum. Moreover, when masks are considered, we ought to concentrate our attention to regions of interest within the source and target images. Let us call $\Omega_{s} \subset \mathbb{R}^{2}$ and $\Omega_{t} \subset \mathbb{R}^{2}$ the regions of interest of the source and target images, respectively. Then, we measure the dissimilarity between the warped source image and the target image by

$$
E_{\text {img }}=\frac{1}{\# \Omega} \sum_{\mathbf{x} \in \Omega}\left(I_{t}(\mathbf{x})-I_{s}(\mathbf{g}(\mathbf{x}))\right)^{2}
$$

where $\Omega=\left\{\mathbf{x} \in \Omega_{t} \cap \mathbb{Z}^{2}: \mathbf{g}(\mathbf{x}) \in \Omega_{s} \cap \mathbb{Z}^{2}\right\}$ defines a mask common to the source and target images, and where $\# \Omega$ is the size of the mask in pixels. Notice that this dissimilarity measure is sensible to linear transformations of the image gray values and, hence, the deformation field found may be different if the gray values in one of the images is transformed. To use this dissimilarity measure it is suggested that both images are brought to a common gray value framework using some normalization procedure [38].

\section{B. Image Representation}

Notice that, in (3), the target image $I_{t}$ is always evaluated at integer positions. However, this is not the case for the source image which needs to be evaluated at possibly noninteger positions $\mathbf{g}(\mathbf{x})$. For this reason, an interpolation scheme must be specified. Cubic B-spline interpolation offers a good tradeoff between accuracy and speed [18], [19]. For carrying out this interpolation, we express the source image as

$$
I_{s}(x, y ; h)=\sum_{k, l \in \mathbb{Z}^{2}} c_{k, l} \beta^{3}\left(\frac{x}{h}-k\right) \beta^{3}\left(\frac{y}{h}-l\right)
$$

where $\beta^{3}(x)$ is the B-spline of degree 3 and where the coefficients $c_{k, l}$ are evaluated according to the least-squares fitting technique described in [39]. $h=2^{0}, 2^{1}, 2^{2}, \ldots$ is a parameter controlling the degree of detail of the representation (smaller values of $h$ provide more detailed representations).

\section{Deformation Representation}

Similarly to [12]-[14], we also express the deformation field as a linear combination of B-splines

$$
\begin{aligned}
\mathbf{g}(\mathbf{x}) & =\mathbf{g}(x, y) \\
& =\left(g_{1}(x, y), g_{2}(x, y)\right) \\
& =\sum_{k, l \in \mathbb{Z}^{2}}\left(\begin{array}{l}
c_{1, k, l} \\
c_{2, k, l}
\end{array}\right) \beta^{3}\left(\frac{x}{s_{x}}-k\right) \beta^{3}\left(\frac{y}{s_{y}}-l\right)
\end{aligned}
$$

where $s_{x}$ and $s_{y}$ are scalars that control the degree of detail of the representation of the deformation field and that need not be a power of 2. Cubic B-splines constitute a Riesz basis of $L_{2}$ and have a fourth order of approximation. Therefore, any sufficiently regular deformation field can be represented with vanishing error using a fine-enough scale. Moreover, by using B-splines of degree 3, the continuity of the derivatives of the deformation is guaranteed up to the second order. Specifically, cubic splines have a fourth order of approximation, meaning that the error between the true deformation $\mathrm{g}_{0}(x)$ and its spline approximation $\mathbf{g}(x)$ is guaranteed to decay like $O\left(s^{p}\right)$ with $s=$ $\max \left(s_{x}, s_{y}\right)$ and $p=\min (r, 4)$, where $r$ (Sobolev regularity) is the maximal degree of differentiability of $\mathbf{g}_{0}(x)$ is the $L_{2}$-sense ([40], Appendix A).

Another advantage of this model is that any affine transformation can be represented by our B-spline deformation [17]. The B-spline of degree 3 fulfills the partition of unity $1=$ $\sum_{k \in \mathbb{Z}} \beta^{3}(x-k)$, and several discrete-moment properties as well, such as $0=\sum_{k \in \mathbb{Z}}(x-k) \beta^{3}(x-k)$. These two properties ensure that $b=\sum_{k \in \mathbb{Z}} b \beta^{3}(x-k)=\sum_{k, l \in \mathbb{Z}^{2}} b \beta^{3}(x-$ $k) \beta^{3}(y-l)$ and that $x=\sum_{k \in \mathbb{Z}} k \beta^{3}(x-k)=\sum_{k, l \in \mathbb{Z}^{2}} k \beta^{3}(x-$ $k) \beta^{3}(y-l)$. Thus, we can express any affine transformation $\mathbf{g}(\mathbf{x})=(\mathbf{A x}+\mathbf{b})$ given by a matrix $\mathbf{A}$ and by a translation $\mathbf{b}$ as

$$
\begin{aligned}
\mathbf{g}(\mathbf{x}) & =\left(\begin{array}{l}
a_{11} x+a_{12} y+b_{1} \\
a_{21} x+a_{22} y+b_{2}
\end{array}\right) \\
& =\sum_{k, l \in \mathbb{Z}^{2}}\left(\begin{array}{c}
c_{1, k, l} \\
c_{2, k, l}
\end{array}\right) \beta^{3}(x-k) \beta^{3}(y-l)
\end{aligned}
$$

with

$$
\left(\begin{array}{l}
c_{1, k, l} \\
c_{2, k, l}
\end{array}\right)=\left(\begin{array}{c}
a_{11} k+a_{12} l+b_{1} \\
a_{21} k+a_{22} l+b_{2}
\end{array}\right)
$$

\section{Landmarks}

We make use of the landmark information in two different ways: first, landmarks are used to impose soft constraints to the deformation field; second, landmarks are used in the initialization stage as described in Section III-F. Soft constraints have been chosen since the landmark position may be contaminated by noise-in other words, trying to satisfy them exactly would be inappropriate. A second reason for using soft landmarks is that a situation can arise where they are distributed in such a way that it is impossible to find a geometrical deformation in the search space (see Section III-C) that completely satisfies all correspondences. 
Let us assume that $N$ pairs of corresponding landmarks $\left(\boldsymbol{\mu}_{s}^{(n)}, \boldsymbol{\mu}_{t}^{(n)}\right), n=1,2, \ldots, N$, are available. Then, we handle the soft landmark constraints by an energy term of the form

$$
E_{\mu}=\frac{1}{N} \sum_{n=1}^{N}\left\|\boldsymbol{\mu}_{t}^{(n)}-\mathrm{g}\left(\boldsymbol{\mu}_{s}^{(n)}\right)\right\|^{2} .
$$

Taking (4) into account, this energy can be computed as

$$
E_{\mu}=\frac{1}{N}\left(\left\|\boldsymbol{\mu}_{t_{1}}-B_{\boldsymbol{\mu}_{s}} \mathbf{c}_{1}\right\|^{2}+\left\|\boldsymbol{\mu}_{t_{2}}-B_{\boldsymbol{\mu}_{s}} \mathbf{c}_{2}\right\|^{2}\right)
$$

where $\boldsymbol{\mu}_{t_{1}}$ and $\boldsymbol{\mu}_{t_{2}}$ are column vectors with all the first and second components, respectively, of the target landmarks, and where $B_{\boldsymbol{\mu}_{s}}$ is a matrix collecting the values of the B-splines of the deformation model evaluated at the source landmarks.

\section{E. Regularization}

The smoothness of the deformation field is a useful regularization term for the minimization problem, especially when little information is available. The traditional regularization term is based on the norm of the second derivative of each of the deformation component separately [24]-[27], as in

$$
\begin{aligned}
E_{\mathrm{D}^{2}}= & \int_{\mathbb{R}^{2}}\left\|\mathrm{D}^{2} g_{1}\right\|^{2} \mathrm{~d} x \mathrm{~d} y+\int_{\mathbb{R}^{2}}\left\|\mathrm{D}^{2} g_{2}\right\|^{2} \mathrm{~d} x \mathrm{~d} y \\
= & \int_{\mathbb{R}^{2}}\left(\partial_{x x} g_{1}\right)^{2} \mathrm{~d} x \mathrm{~d} y+\int_{\mathbb{R}^{2}}\left(\partial_{y y} g_{1}\right)^{2} \mathrm{~d} x \mathrm{~d} y \\
& +2 \int_{\mathbb{R}^{2}}\left(\partial_{x y} g_{1}\right)^{2} \mathrm{~d} x \mathrm{~d} y \\
& +\int_{\mathbb{R}^{2}}\left(\partial_{x x} g_{2}\right)^{2} \mathrm{~d} x \mathrm{~d} y+\int_{\mathbb{R}^{2}}\left(\partial_{y y} g_{2}\right)^{2} \mathrm{~d} x \mathrm{~d} y \\
& +2 \int_{\mathbb{R}^{2}}\left(\partial_{x y} g_{2}\right)^{2} \mathrm{~d} x \mathrm{~d} y .
\end{aligned}
$$

There, $D$ is the total differential operator, $\left(\partial_{u v} g\right)^{2}$ is the square of the second derivative of the function $g$ with respect to $u$ and $v$. This regularization term is related to the stress of a stretched elastic material. Thin-plate splines are defined upon the minimization of this energy [28]. This regularizing term treats each component of the deformation field independently and is not imposing a truly smooth condition to a vector field. The authors of [29] studied the interpolation of wind velocity fields using 2 -D vector splines and proposed the following two regularizing terms that fully exploit the vectorial nature of the data:

$$
\begin{aligned}
E_{\text {rough }}= & w_{d} E_{\text {div }}+w_{r} E_{\text {rot }} \\
= & w_{d} \int_{\mathbb{R}^{2}}\|\nabla \operatorname{divg}\|^{2} \mathrm{~d} x \mathrm{~d} y \\
& +w_{r} \int_{\mathbb{R}^{2}}\|\nabla \operatorname{rotg}\|^{2} \mathrm{~d} x \mathrm{~d} y
\end{aligned}
$$

where

$$
\operatorname{divg}=\partial_{x} g_{1}+\partial_{y} g_{2}
$$

represents the divergence of the 2-D vector field $\mathrm{g}$, and where

$$
\operatorname{rotg}=-\partial_{y} g_{1}+\partial_{x} g_{2}
$$

represents the length of the unique component of the curl of $\mathbf{g}$, and where $\nabla f=\left(\partial_{x} f, \partial_{y} f\right)$ is the gradient of the scalar function $f$. The divergence of a vector field is related to the existence in the vector field of sinks and sources, while the curl is related to the rotation of a "differential twig" within the field [41]. Therefore, the proposed regularization penalizes changes in the local structure of the divergence and curl. However, it has been shown in [29] that, for any two functions in the BeppoLevi space of order 2, the following relation holds, $\forall a, b, c, d \in$ $\{x, y\}$ :

$$
\int_{\mathbb{R}^{2}} \partial_{a b} g_{1} \partial_{c d} g_{2} \mathrm{~d} x \mathrm{~d} y=\int_{\mathbb{R}^{2}} \partial_{a d} g_{1} \partial_{c b} g_{2} \mathrm{~d} x \mathrm{~d} y .
$$

Therefore, (6) is a special case of (7) when $w_{d}=w_{r}$. This regularization term has been successfully applied to a number of landmark-only applications in image processing [10], [23], [30]. More precisely, when $w_{d}=w_{r}=1$, then $E_{\text {rough }}$ simplifies to

$$
\begin{aligned}
E_{\mathrm{div}}+E_{\text {rot }}= & E_{\mathrm{D}^{2}}+\left(2 \int_{\mathbb{R}^{2}} \partial_{x x} g_{1} \partial_{x y} g_{2} \mathrm{~d} x \mathrm{~d} y\right. \\
& +2 \int_{\mathbb{R}^{2}} \partial_{x y} g_{1} \partial_{y y} g_{2} \mathrm{~d} x \mathrm{~d} y \\
& -2 \int_{\mathbb{R}^{2}} \partial_{x y} g_{1} \partial_{x x} g_{2} \mathrm{~d} x \mathrm{~d} y \\
& \left.-2 \int_{\mathbb{R}^{2}} \partial_{y y} g_{1} \partial_{x y} g_{2} \mathrm{~d} x \mathrm{~d} y\right)
\end{aligned}
$$

and all the terms in parenthesis cancel one another. It should be noticed that this cancellation does not necessarily occur if the integration domain is finite.

A first advantage of minimizing $E_{\text {rough }}$, which is a measure of the true roughness of the deformation, is that it results in a deformation that is truly smooth because it includes the cross terms that were ignored in $E_{\mathrm{D}^{2}}$. A second advantage of this regularization is that it gives the user more freedom to express her a priori knowledge about the underlying deformation.

For instance, let us suppose that the underlying deformation field takes the form $\mathbf{g}(\mathbf{x})=\left(x, g_{2}(x)+K y\right)$; then, we have that $0=\int_{\mathbb{R}^{2}}\|\nabla \operatorname{divg}(\mathbf{x})\|^{2} \mathrm{~d} x \mathrm{~d} y \ll \int_{\mathbb{R}^{2}}\|\nabla \operatorname{rotg}(\mathbf{x})\|^{2} \mathrm{~d} x \mathrm{~d} y=$ $\int_{\mathbb{R}^{2}}\left(\partial_{x x} g_{2}\right)^{2} \mathrm{~d} x \mathrm{~d} y$. In this situation, it is desirable to put much more weight on the regularization term based on the divergence of the deformation field than on the one based on its curl. We show in Section IV-A2 that this kind of regularization is very appropriate for the correction of the "smile" effect in electrophoresis.

Alternatively, we discuss now the converse case where it is desirable to emphasize more $w_{r}$ than $w_{d}$, which illustrates the fact that other a priori structures can easily be introduced through the regularization terms. For instance, we might know that there are only horizontal deformations and that they are more important as we go away from a vertical symmetry axis situated at $x=x_{0}$. Then, a good model for this deformation would be $\mathbf{g}(\mathbf{x})=\left(x+\left(x-x_{0}\right) g_{1}(y), y\right)$ and, consequently

$$
\begin{aligned}
\int_{\mathbb{R}^{2}}\left(\partial_{y} g_{1}\right)^{2} \mathrm{~d} x \mathrm{~d} y & =\int_{\mathbb{R}^{2}}\|\nabla \operatorname{divg}(\mathbf{x})\|^{2} \mathrm{~d} x \mathrm{~d} y \\
& <\int_{\mathbb{R}^{2}}\|\nabla \operatorname{rotg}(\mathbf{x})\|^{2} \mathrm{~d} x \mathrm{~d} y \\
& =\int_{\mathbb{R}^{2}}\left(\left(\partial_{y} g_{1}\right)^{2}+\left(\left(x-x_{0}\right) \partial_{y y} g_{1}\right)^{2}\right) \mathrm{d} x \mathrm{~d} y .
\end{aligned}
$$

Moreover, let us assume that our deformation is radially isotropic (i.e., that it can be expressed in polar coordinates as $\mathbf{g}(r, \phi)=g_{r}(r) \mathbf{r}$, where $(r, \phi)$ are the polar coordinates 
of the point $\mathbf{x}$, and where $\mathbf{r}$ is the radial vector). Using the corresponding polar formullae for the curl, divergence, and gradient, one can show that

$$
\begin{aligned}
\int_{0}^{\infty} & \int_{0}^{2 \pi}\left(\partial_{r r} g_{r}+\frac{\partial_{r} g_{r}}{r}-\frac{g_{r}}{r^{2}}\right)^{2} \mathrm{~d} \phi \mathrm{d} r \\
& =\int_{0}^{\infty} \int_{0}^{2 \pi}\|\nabla \operatorname{divg}(r, \phi)\|^{2} \mathrm{~d} \phi \mathrm{d} r \\
& \gg \int_{0}^{\infty} \int_{0}^{2 \pi}\|\nabla \operatorname{rotg}(r, \phi)\|^{2} \mathrm{~d} \phi \mathrm{d} r=0
\end{aligned}
$$

which suggests setting $w_{r}$ very large.

Since the deformation field is expressed in terms of B-splines, the energy term (7) can be computed in a very efficient way. Concentrating the evaluation of the roughness energy only in the region $X_{t}=\left[0, X_{\mathrm{dim}}\right] \times\left[0, Y_{\mathrm{dim}}\right]$ where the target image is defined, we observe that all integrals are of the form

$$
\int_{X_{t}} \frac{\partial^{q_{1}+q_{2}} g_{i}}{\partial x^{q_{1}} \partial y^{q_{2}}} \frac{\partial^{q_{3}+q_{4}} g_{j}}{\partial x^{q_{3}} \partial y^{q_{4}}} \mathrm{~d} x \mathrm{~d} y
$$

where $i, j \in\{1,2\}$. Using (4), we show that

$$
\begin{aligned}
\int_{X_{t}} & \frac{\partial^{q_{1}+q_{2}} g_{i}}{\partial x^{q_{1}} \partial y^{q_{2}}} \frac{\partial^{q_{3}+q_{4}} g_{j}}{\partial x^{q_{3}} \partial y^{q_{4}}} \mathrm{~d} x \mathrm{~d} y \\
= & \sum_{k, l, m, n}\left(c_{i, k, l} \eta_{q_{1}, q_{3}}\left(k h, m h, X_{\mathrm{dim}}\right)\right. \\
& \left.\times \eta_{q_{2}, q_{4}}\left(l h, n h, Y_{\mathrm{dim}}\right) c_{j, m, n}\right) \\
= & \mathbf{c}_{i}^{\top} \mathbf{R}_{q_{1}, q_{2}, q_{3}, q_{4}} \mathbf{c}_{j}
\end{aligned}
$$

where $\mathbf{c}_{i}$ and $\mathbf{c}_{j}$ are column vectors with all the B-spline coefficients corresponding to the deformation components $g_{i}$ and $g_{j}$ respectively, $\mathbf{R}_{q_{1}, q_{2}, q_{3}, q_{4}}$ is a matrix with all the products $\eta_{q_{1}, q_{3}}\left(k h, m h, X_{\mathrm{dim}}\right) \eta_{q_{2}, q_{4}}\left(l h, n h, Y_{\mathrm{dim}}\right)$ in an appropriate order, and

$$
\eta_{q_{1}, q_{2}}\left(s_{1}, s_{2}, L\right)=\int_{0}^{L} \alpha_{q_{1}}\left(x-s_{1}\right) \alpha_{q_{2}}\left(x-s_{2}\right) \mathrm{d} x
$$

with

$$
\alpha_{q}(x)=\frac{\partial^{q} \beta^{3}(x)}{\partial x^{q}}
$$

Since B-splines are piecewise polynomials, the $\eta$ integrals can be precomputed exactly using closed formulæ. Therefore, the evaluation of the roughness energy reduces to a sum of three bilinear forms. Making $\mathbf{R}_{q_{1}, q_{2}}=\mathbf{R}_{q_{1}, q_{2}, q_{1}, q_{2}}$ and using (8), we rewrite (7) as

$$
\begin{aligned}
E_{\text {rough }} & \mathbf{c}_{1}^{\top} \underbrace{\left(w_{d}\left(\mathbf{R}_{20}+\mathbf{R}_{11}\right)+w_{r}\left(\mathbf{R}_{11}+\mathbf{R}_{02}\right)\right)}_{\mathbf{P}_{11}} \mathbf{c}_{1} \\
& +\mathbf{c}_{1}^{\top} \underbrace{2\left(w_{d}\left(\mathbf{R}_{2011}+\mathbf{R}_{1102}\right)-w_{r}\left(\mathbf{R}_{1102}+\mathbf{R}_{2011}\right)\right)}_{\mathbf{P}_{12}} \mathbf{c}_{2} \\
& +\mathbf{c}_{2}^{\top} \underbrace{\left(w_{d}\left(\mathbf{R}_{02}+\mathbf{R}_{11}\right)+w_{r}\left(\mathbf{R}_{11}+\mathbf{R}_{20}\right)\right)}_{\mathbf{P}_{22}} \mathbf{c}_{2} \\
= & \mathbf{c}_{1}^{\top} \mathbf{P}_{11} \mathbf{c}_{1}+\mathbf{c}_{1}^{\top} \mathbf{P}_{12} \mathbf{c}_{2}+\mathbf{c}_{2}^{\top} \mathbf{P}_{22} \mathbf{c}_{2} .
\end{aligned}
$$

We use this latter expression as regularizing term. Its computation is very fast and efficient since the matrices $\mathbf{P}_{i j}$ can be precomputed. Furthermore, this expression allows an easy computation of the derivatives of the regularization term, as will be shown in Section III-I.

\section{F. Landmark-Only Registration}

To obtain that only the landmarks are used to register the two images, we set $w_{i}=0$ in (1). Then, the optimal deformation coefficients $\mathbf{c}_{1}$ and $\mathbf{c}_{2}$ can be computed analytically through the equations $\left(\partial E / \partial \mathbf{c}_{1}\right)=(\partial E / \partial \mathbf{c})_{2}=\mathbf{0}$. These two equations yield a linear equation system characterized by $\mathbf{Q c}=2 B_{\boldsymbol{\mu}_{s}}^{\top} \boldsymbol{\mu}_{t}$, with $\mathbf{c}=\left(\mathbf{c}_{1}, \mathbf{c}_{2}\right)$ and with

$$
\begin{aligned}
& \mathbf{Q}=2 \omega_{\mu}\left(\begin{array}{cc}
B_{\boldsymbol{\mu}_{s}^{\top} B_{\boldsymbol{\mu}_{s}}} & 0 \\
0 & B_{\boldsymbol{\mu}_{s}}^{\top} B_{\boldsymbol{\mu}_{s}}
\end{array}\right) \\
&+\left(\begin{array}{cc}
\mathbf{P}_{11}+\mathbf{P}_{11}^{\top} & \mathbf{P}_{12} \\
\mathbf{P}_{12}^{\top} & \mathbf{P}_{22}+\mathbf{P}_{22}^{\top}
\end{array}\right) .
\end{aligned}
$$

The matrix $B_{\boldsymbol{\mu}_{s}}$ was introduced in Section III-D, while the matrices $\mathbf{P}_{i j}$ were defined in Section III-E. The vector $\boldsymbol{\mu}_{t}$ is a column vector with all the first components of the target landmarks in front, and then the second components of the target landmarks.

This is the solution that best fits the landmarks for a given number of deformation coefficients, while satisfying the regularization constraints. This regularized landmark-only registration generalizes some of the landmark-only registration algorithms by explicit considering the smoothness constraints. We use the landmark-only solution to initialize our iterative optimizer (see Section III-H).

\section{G. Multiresolution Strategy}

As in other B-spline-based elastic-registration algorithms, we perform the search of the deformation in a multiresolution fashion. We start with the coarsest representation of the deformation field and the images. The optimization procedure described in Section III-H is applied until convergence to estimate the deformation field at this level. The optimizer is first initialized by the analytical solution given in (10). Then, the resolution in the deformation field and in the image is increased until the finest levels of details are reached. To increase the resolution in the deformation field, we use the expansion operator described in [37]; this expanded deformation is then used as initialization at the next stage.

\section{H. Optimizer}

We use an optimization algorithm that is inspired by the Levenberg-Marquardt nonlinear regression [34]. This algorithm achieves a gradual transition between quasi-Newton and gradient-descent steps. The specific tradeoff depends on the goodness of a local model of the function being minimized. Briefly, this method updates the deformation coefficients $\mathbf{c}=\left(\mathbf{c}_{1}, \mathbf{c}_{2}\right)$ in the iterative fashion $\mathbf{c}^{(k+1)}=\mathbf{c}^{(k)}+\Delta \mathbf{c}^{(k)}$, where $\Delta \mathbf{c}$ is the solution of the equation system $\tilde{\mathbf{H}} \Delta \mathbf{c}^{(k)}=\nabla E\left(\mathbf{c}^{(k)}\right)$. There, $\nabla E\left(\mathbf{c}^{(k)}\right)$ is the gradient of the energy with respect to the deformation coefficients evaluated at $\mathbf{c}^{(k)}$, and $\tilde{\mathbf{H}}$ is a modified version of the Hessian $\mathbf{H}$ such that the component $[\tilde{\mathbf{H}}]_{i j}=\left(1+\lambda \delta_{i j}\right)[\mathbf{H}]_{i j}$, where $\delta_{i j}=1-|\operatorname{sign}(i-j)|$ is Kronecker's delta. When $\lambda$ is large the step is more steepest-descent-like while, for small values of $\lambda$, it is more Newton-like. 
$\lambda$ is adaptively modified according to successes or failures of $\mathbf{c}^{(k)}$ to minimize the given energy.

The authors of [34] used a diagonal approximation to the true Hessian based only on first derivatives of the energy function. This is a fair approximation close to the minimum. However, the procedure can benefit from a BFGS estimation of the Hessian [35]. An advantage of the BFGS approximation to the Hessian is that it is always positive semi-definite, which is a mandatory requirement for the stability of a quasi-Newton optimization algorithm. BFGS results in the following iterative approximation to the Hessian:

$$
\begin{aligned}
\Delta \mathbf{c}^{(k)} & =\mathbf{c}^{(k+1)}-\mathbf{c}^{(k)} \\
\Delta \mathbf{f}^{(k)} & =\nabla E\left(\mathbf{c}^{(k+1)}\right)-\nabla E\left(\mathbf{c}^{(k)}\right) \\
\Delta \mathbf{H c}^{(k)} & =\mathbf{H}^{(k)} \Delta \mathbf{c}^{(k)} \\
\mathbf{H}^{(k+1)}= & \mathbf{H}^{(k)}+\frac{\Delta \mathbf{f}^{(k)} \otimes \Delta \mathbf{f}^{(k)}}{\left\langle\Delta \mathbf{f}^{(k)}, \Delta \mathbf{c}^{(k)}\right\rangle} \\
& -\frac{\Delta \mathbf{H} \mathbf{c}^{(k)} \otimes \Delta \mathbf{H} \mathbf{c}^{(k)}}{\left(\Delta \mathbf{c}^{(k)}\right)^{\top} \mathbf{H}^{(k)} \Delta \mathbf{c}^{(k)}}
\end{aligned}
$$

where $\mathbf{u} \otimes \mathbf{v}=\mathbf{u v}^{\top}$ and $\langle\mathbf{u}, \mathbf{v}\rangle=\mathbf{u}^{\top} \mathbf{v}$. The Hessian estimate is initialized by the diagonal approximation of [34] and is updated only on successful estimates $\mathbf{c}^{(i)}, k \neq i$. For keeping the semipositive-definite quality of the estimate, this update can only be performed if $\left\langle\Delta \mathbf{f}^{(k)}, \Delta \mathbf{c}^{(k)}\right\rangle>0$ [42, Chapter 9].

\section{Explicit Derivatives}

Our optimizer makes extensive use of the derivatives of the different energy terms. Since the images and the deformation field are expressed in B-splines, these derivatives can be computed exactly.

Data term: from (3), it can be easily shown that the derivative of $E_{\text {img }}$ with respect to any of the deformation coefficients defining the first component of the deformation field $g$ is

$$
\begin{aligned}
& \frac{\partial E_{\text {img }}}{\partial c_{1, k, l}}=-2 \sum_{\mathbf{x} \in \Omega}\left(\left(I_{t}(\mathbf{x})-I_{s}(\mathbf{g}(\mathbf{x}))\right) \frac{\partial I_{s}(\mathbf{g}(\mathbf{x}))}{\partial g_{1}(\mathbf{x})}\right. \\
&\left.\times \beta^{3}\left(\frac{x}{s}-k\right) \beta^{3}\left(\frac{y}{s}-l\right)\right)
\end{aligned}
$$

where $\left(\partial I_{s}(\mathbf{g}(\mathbf{x}))\right) /\left(\partial g_{1}(\mathbf{x})\right)$ is the derivative of the source image in the $x$ direction evaluated at the point $\mathbf{g}(\mathbf{x})$. The derivative with respect to one of the coefficients of the second component of the deformation model is analog to this one.

Landmarks term: it can be easily shown that the derivatives of the landmark energy (5) are given by

$$
\begin{aligned}
& \frac{\partial E_{\mu}}{\partial \mathbf{c}_{1}}=-2 B_{\boldsymbol{\mu}_{s}}^{\top}\left(\boldsymbol{\mu}_{t_{1}}-B_{\boldsymbol{\mu}_{s}} \mathbf{c}_{1}\right) \\
& \frac{\partial E_{\mu}}{\partial \mathbf{c}_{2}}=-2 B_{\boldsymbol{\mu}_{s}}^{\top}\left(\boldsymbol{\mu}_{t_{2}}-B_{\boldsymbol{\mu}_{s}} \mathbf{c}_{2}\right) .
\end{aligned}
$$

Regularization term: this term is computed as indicated by (9). The derivatives of these bilinear forms are easy to compute and yield

$$
\begin{aligned}
& \frac{\partial E_{\text {rough }}}{\partial \mathbf{c}_{1}}=\left(\mathbf{P}_{11}+\mathbf{P}_{11}^{\top}\right) \mathbf{c}_{1}+\mathbf{P}_{12} \mathbf{c}_{2} \\
& \frac{\partial E_{\text {rough }}}{\partial \mathbf{c}_{2}}=\left(\mathbf{P}_{22}+\mathbf{P}_{22}^{\top}\right) \mathbf{c}_{1}+\mathbf{P}_{12}^{\top} \mathbf{c}_{1} .
\end{aligned}
$$

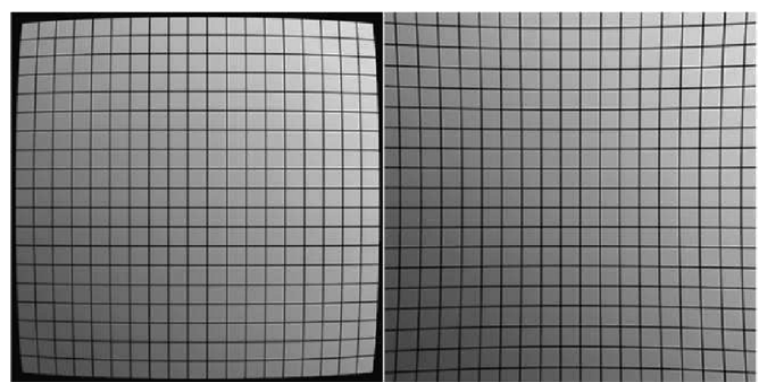

Fig. 1. Cartesian grid after a barrel deformation (left) and a pincushion deformation (right) has been applied.

\section{RESULTS}

We performed many experiments in order to test the ability of our algorithm to correct for deformations. We present computersimulated experiments in Section IV-A, while we present real cases in Section IV-B where we investigate the performance of our method in the context of experimental 2-D electrophoretic gels and confocal scanning microscopy images of fly embryos.

\section{A. Controlled Data}

1) Accuracy: We have tested the accuracy of our algorithm by using computer simulations: an image is subjected to a known deformation $\mathrm{g}^{*}$; then, the registration algorithm is used to restore the original image from the warped one. The deformation given by the registration algorithm is g. The accuracy of this process is measured by the "warping index" defined as $\varpi=\sqrt{\left(1 /\left\|X_{s}\right\|\right) \sum_{\mathbf{x} \in X_{s}}\left\|\mathbf{x}-\mathbf{g}\left(\mathbf{g}^{*}(\mathbf{x})\right)\right\|^{2}}$. This figure of merit is standard in the field [12], [34].

The true deformations were picked from a deformation space that cannot be represented exactly by B-splines. The goal is to test the algorithm performance in a challenging situation that does not allow for an exact registration since this would bias our estimate of the accuracy. In particular, the barrel/pincushion distortion [43] was used. This is a distortion which is frequently present in imaging devices such as cameras, light and electron microscopes, monitors. If the input coordinate $\mathbf{x}_{\text {in }}$ is normalized to lie in $[-1,1] \times[-1,1]$, and if $r_{\text {in }}$ is the radius of its polar expression in this coordinate system, then the output radius produced by the distortion is

$$
r_{\text {out }}=r_{\text {in }}\left(1+K_{1} r_{\text {in }}^{2}+K_{2} r_{\text {in }}^{4}\right) \text {. }
$$

The nature of barrel or pincushion deformation is given by the signs of $K_{1}$ and $K_{2}$. An identity transformation is provided by $K_{1}=K_{2}=0$.

We generated 30 random barrel/pincushion transformations by adding zero-mean Gaussian noise with standard deviation 0.05 to $K_{1}$ and with standard deviation 0.025 to $K_{2}$. We give in Fig. 1 two deformation examples, while we show in Fig. 2 the test image used for the simulations. Our algorithm was used with neither regularization nor landmarks $\left(w_{i}=1 ; w_{\mu}=w_{d}=\right.$ $\left.w_{r}=0\right)$. The deformation pyramid had 2 levels, and the image pyramid had 3 levels. (These level counts include the finest level.) The initial warping index of the unregistered images was $14.00 \pm 22.08$ pixel, and the final warping index (after registration) was $0.06 \pm 0.05$ pixel.

2) Regularization Versatility: Electrophoresis has de facto become the standard procedure for separating proteins within 


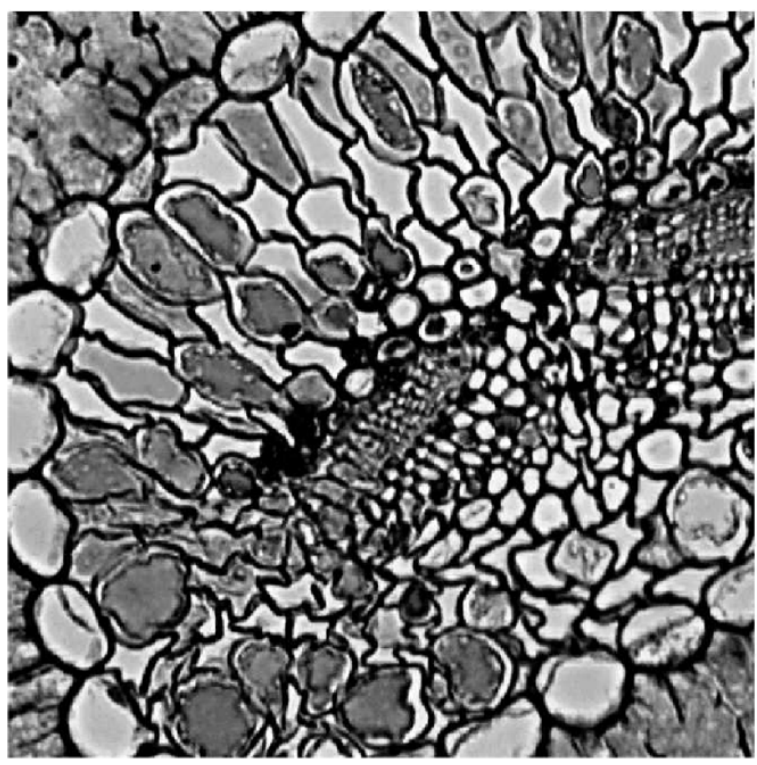

Fig. 2. Pine-needle cross section taken by optical microscopy. The size of this image is $512 \times 512$.

cells and tissues. A purified solution of proteins is injected into a polyacrylamide gel. Then, proteins are separated according to their mass by electrophoresis in the presence of SDS and according to their electric charge by a $\mathrm{pH}$ gradient. Finally, a dye is applied with a selective affinity toward specific components of the proteins. The result is a bidimensional (mass and charge) image where small spots reveal the presence of a protein with a certain mass and charge in the initial solution. We show in the left part of Fig. 3 an example of an electrophoretic gel.

A common deformation in gel electrophoresis is known as the "smile effect" (dye-front deformation, [44]). After the coordinates have been normalized to lie in $[0,1] \times[0,1]$, its simplified deformation model is given by

$$
\mathbf{g}(\mathbf{x})=\left(x, K y-s_{0}\left(1-(1-2 x)^{2}\right)\right)
$$

where $K$ is a global scale-reduction factor on the vertical direction, and where $s_{0}$ is the maximum deformation in the middle of the image. For this deformation, $\|\nabla \operatorname{divg}(\mathbf{x})\|^{2}=0$ and $\|\nabla \operatorname{rotg}(\mathbf{x})\|^{2}=\left(8 s_{0}\right)^{2}$. We give in the right part of Fig. 3 an example of this deformation with a factor reduction of $5 \%$ and a maximum central shift of $14 \%$. (These deformation parameters are typical for comparing different image registration methods for 2-D gels [45].) The unregularized problem converges to an unsatisfactory solution. The problem is that the resulting deformation field is not regular, as can be seen in Fig. 4. Furthermore, we were unable to produce a satisfactory solution if we restricted ourselves to enforce $w_{d}=w_{r}$. However, successful unwarping is achieved with $w_{d}=4$ and $w_{r}=0$ (with $w_{i}=1$ and $w_{\mu}=0$ ). The deformation pyramid had 2 levels, and the image pyramid had 3 levels. We report in Fig. 5 the evolution of the measure of dissimilarity $E_{\mathrm{img}}$, along with the number of iterations for the unregularized and the regularized solution.

3) Landmark Usefulness: Most of the works in electrophoresis image registration is based on landmark matching or spot matching [7], [11], [46]-[49]. On one hand, matching spots is a more complex problem than matching landmarks since their correspondence is unknown and since the number

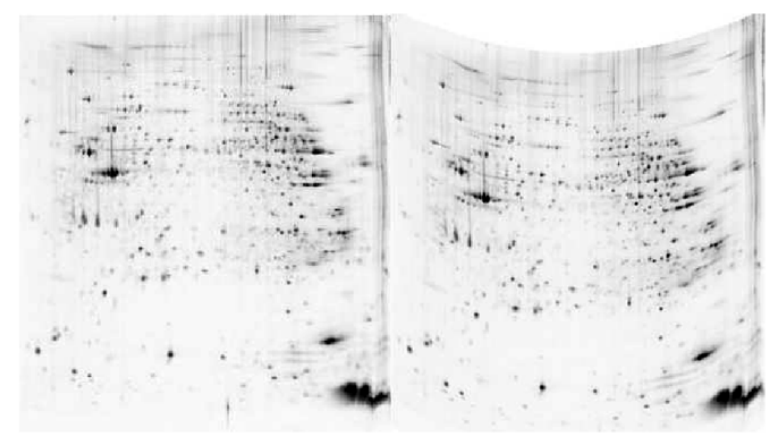

Fig. 3. Pair of corresponding 2-D gels related by a "smile" transformation. The image on the left is unprocessed data, while the image on the right was simulated.

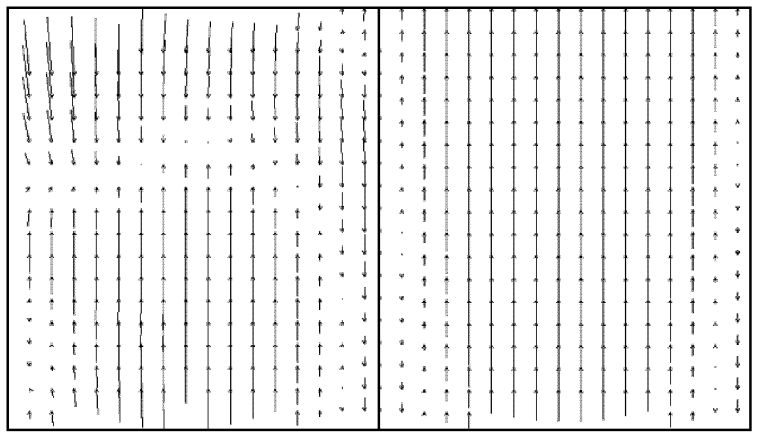

Fig. 4. Left: Deformation field of the unregularized registration of the 2-D gel shown in Fig. 3. Right: True deformation field.

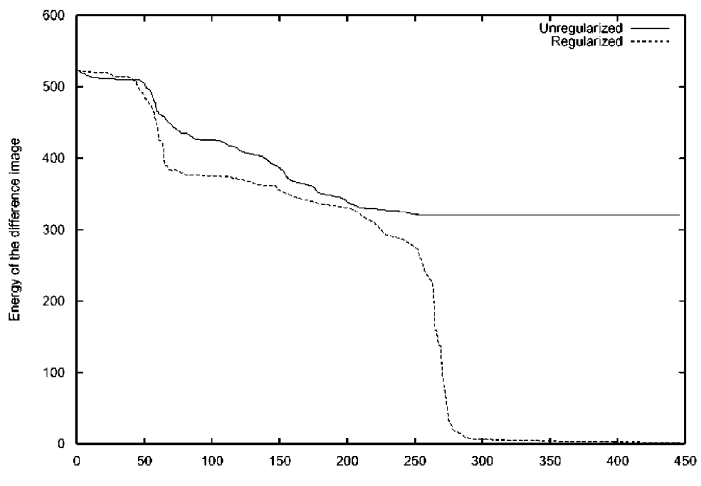

Fig. 5. Plot of the measure of dissimilarity versus the number of iterations for the unregularized and regularized registration of the 2-D gel shown in Fig. 3.

of spots in both images may differ. On the other hand, the spot extraction can be done fully automatically while landmarks require user input.

To the best of our knowledge, the only registration methods that work directly on the electrophoretic image information can be found in [33] and [50]-[52]. The method in [50] solves a differential equation for the transformation field after smartly deriving an image formation model. The method in [33] can also handle landmarks, image intensities and a regularization term. The elastic transformation field is not parametrized and the regularization term is based on its quadratic energy. The methods in [51], [52] produce a piecewise-bilinear mapping. In [51], the grid is generated by a Delaunay triangulation, while a regular grid is deformed to adapt to the image characteristics in [52]. 


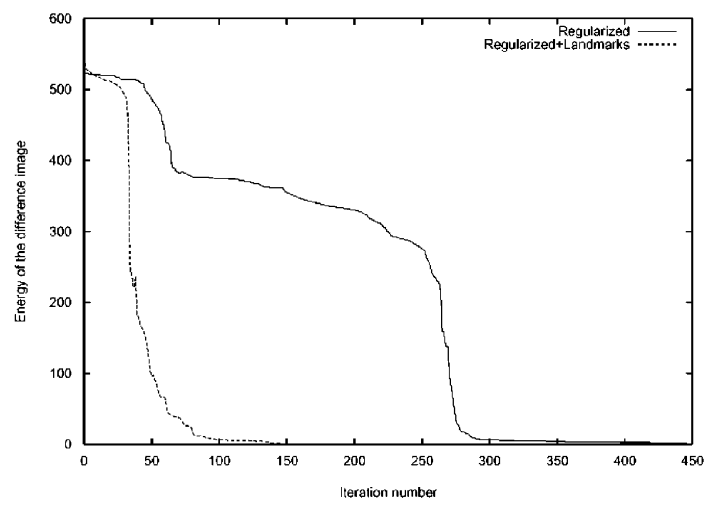

Fig. 6. Plot of the measure of dissimilarity versus the number of iterations for the regularized and regularized+landmarks registration of the 2-D gel shown in Fig. 3.

None of these two algorithms benefits from landmarks or from regularization in the optimization process, a limitation that we overcome with the algorithm proposed in this paper.

In our algorithm, the main effect of the landmark contribution is to accelerate the convergence process substantially, as can be seen in Fig. 6 for the "smile" distorted image of Fig. 3. In this example, we interactively provided four landmarks, one on each quadrant of the image; the landmark weight was $w_{\mu}=1$.

Although our algorithm is capable of combining landmarks and image data, some approaches work with landmarks only. In the next experiment, we adjust the number of perfect landmarks necessary to achieve about the same registration accuracy as when the data term is included in the minimization problem. We generated 30 random "smile" deformations. The reduction factor was uniformly distributed between $0 \%$ and $5 \%$, while the maximum central shift was uniformly distributed between $0 \%$ and $15 \%$. The deformation field was always represented with $4 \times 4$ grid points.

For the experiment taking into account the content of the images, we put a random landmark in each of the four image quadrants. To simulate the uncertainty on the landmarks when they are manually selected, we added random Gaussian noise with zero mean and standard deviation 1 pixel to the $x$ and $y$ components of each of these four landmarks. The deformation field had two pyramid levels, and the image pyramid three. The weights of the data term and the landmarks were both set to $w_{i}=w_{\mu}=1$, while the weight of the regularizing term based on the divergence was set to $w_{d}=4$. We show the achieved accuracy in the first line of Table I. The initial warping index for this experiment was $20.52 \pm 12.80$.

For the landmark-only experiments (remaining lines of Table I), we generated $N$ random landmarks. The first four landmarks were distributed as described for the previous experiment, while the rest of the landmarks were randomly distributed all over the image. No noise was added to the landmark positions, assuming that the automatic landmark-selection algorithm performed a perfect job. We conducted two experiments: one with regularization $\left(w_{\text {div }}=4, w_{\text {rot }}=0\right)$, and another one without regularization, discounting the implicit regularization present in the $4 \times 4$ B-spline-based deformation model $\left(w_{\text {div }}=w_{\text {rot }}=0\right)$. We show in Table $\mathrm{I}$ the achieved accuracy and the number of landmarks needed so that the
TABLE I

FINAL WARPING INDEX $\varpi$ WHEN THE IMAGE INFORMATION IS CONSIDERED $\left(w_{i} \neq 0\right)$ AND THE PRoblem Is Regularized $\left(w_{d} \neq 0\right)$, WHEN THE IMAGE Information Is DisRegarded $\left(w_{i}=0\right)$ BUt THE PROBlem Is StILL REGULARIZED, AND WHEN BOTH IMAGE DATA AND REGULARIZATION ARE IGNORED (LANDMARK-ONLY, $w_{i}=w_{d}=w_{r}=0$ ). IN EACH CASE, $N$ LANDMARKS ARE ALWAYS CONSIDERED $\left(w_{\mu} \neq 0\right)$

\begin{tabular}{r|r|r|r|r|r}
\hline \hline$w_{i}$ & $w_{\mu}$ & $w_{d}$ & $w_{r}$ & $N$ & $\varpi$ \\
\hline 1 & 1 & 1 & 0 & 4 & $0.060 \pm 0.059$ \\
0 & 1 & 4 & 0 & 90 & $0.090 \pm 0.056$ \\
0 & 1 & 0 & 0 & 190 & $0.099 \pm 0.070$ \\
\hline \hline
\end{tabular}

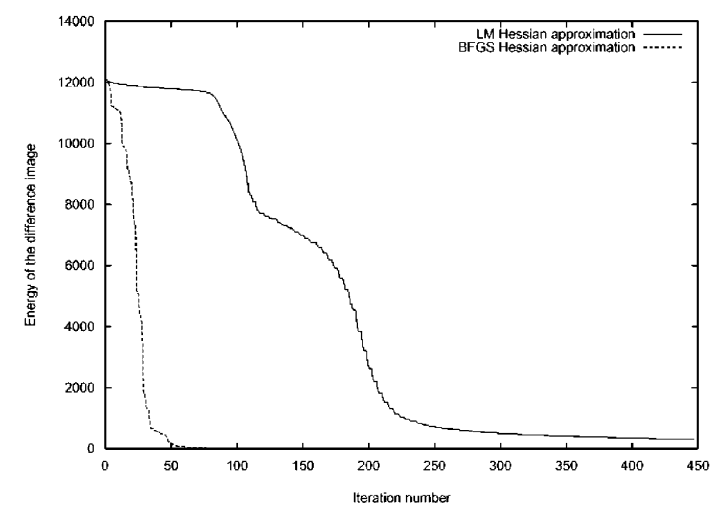

Fig. 7. Plot of the measure of dissimilarity versus the number of iterations for a $5^{\circ}$ rotation of the pine image in Fig. 2 using the Hessian estimation proposed in [34] and the BFGS estimate proposed in this article.

hypothesis that the average final warping index is the same for the three experiments cannot be rejected with a confidence of $99 \%$.

4) Hessian Estimate: We now compare the effect of the Hessian estimate proposed in [34] to that of the Hessian estimate provided by BFGS. To perform this comparison, we registered a rotated version $\left(5^{\circ}\right)$ of the pine-needle image of Fig. 2 with the same image without rotation. We did not take benefit of multiresolution in this experiment; also, we set $w_{\mu}=0$ (landmark-less registration) and $w_{d}=w_{r}=0$. We show in Fig. 7 the value of the measure of dissimilarity as a function of the iteration number.

\section{B. Experimental Data}

1) Electrophoretic Gels: We applied our registration algorithm to the alignment of experimental 2-D electrophoretic gels. We performed experiments in which we used pair of images such as those in Fig. 8. These images represent the protein content of a solution under different biological conditions. The main difference with respect to the experiments of Section IV-A is that the images in a pair do not show the same information since it is in their very difference that the useful information lies. In other words, we are interested to know which proteins are present in one gel and absent from the other.

To preprocess the images, we compensated for background inhomogeneities by using a rolling ball of size 50 pixel [53]. The registration conditions were $N=4, w_{i}=w_{\mu}=1$, and $w_{d}=w_{r}=0$. We show in Fig. 8 the resulting registered image, the initial difference image, the final difference image, and the deformation field. The algorithm was also tested on 


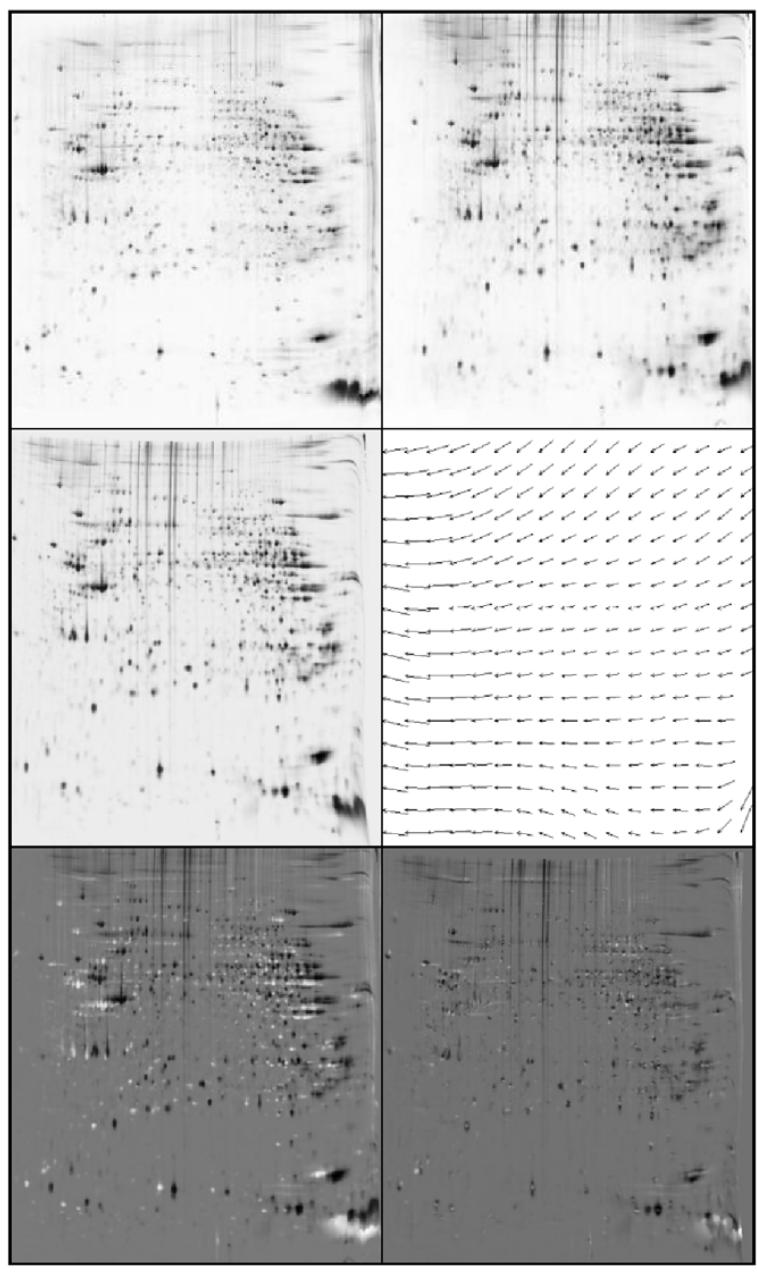

Fig. 8. From top to down, left to right: target image, source image, registered image, recovered deformation field, difference image before registration, difference image after registration.

many other pairs of images in collaboration with the Swiss Institute of Bioinformatics at Geneva. The expert evaluation of the registered images was quite positive in all cases.

2) Confocal Scanning Microscopy: The ability to visualize and quantify molecules in cells with high spatial and temporal resolution is essential for understanding biological systems. Recently, this has become possible due to the advent of confocal scanning microscopy and fluorescent labeling of proteins and RNA. In the present application, the aim is to study the spatial distribution of the genomic regulatory network that coordinates patterns of gene expression, the cell-type specification and tissue differentiation during the evolution of the embryo can be analyzed.

We applied our algorithm to the registration of the confocal scanning microscopy images of Drosophila melanogaster embryos published in [54]. These images are instrumental in the study of the segment differentiation in insects during the zygote development, and of the identification of the genomic regulator that causes it. ${ }^{1}$ These images reveal the expression patterns of

${ }^{1}$ The Department of Computational Biology at the Center for Advanced Studies of Saint Petersburg State Polytechnical University holds a database with hundreds of images of fly embryos at different developmental stages. Our algorithm helps to build accurate bidimensional genomic maps of the embryos at each stage. three genes, each one stained with a different fluorescent dye, as illustrated in the upper part of Fig. 9. Each gene is acquired with a different color. By experimental design, one of the genes remains the same in all embryos; the successful registration of this gene allows the co-registration of the other two for each fly. Hence, the registration algorithm must be performed on one of the color channels and must be applied to the other two.

After registering all embryos, a precise map of the gene expression can be built. To proceed with registration, we set $w_{i}=$ $1, w_{\mu}=0$, and $w_{r}=w_{d}=1$. The deformation pyramid had 2 levels, while the image pyramid had 3. We illustrate in Fig. 9 the expression pattern of the eve gene of two embryos, the registered image, the initial difference image, the final difference image, and the deformation field. This is the first attempt registering this data in two dimensions; previous approaches were always unidimensional.

\section{DISCUSSION}

B-spline-based deformation models reach a high accuracy when correcting for random deformations generated using wavelets [12]. They are also suitable for the type of deformations encountered with Magnetic Resonance Imaging [14]. In the present work, we have successfully extended their use to other applications as well: barrel/pincushion distortions and dye-front curvature ("smile" effect) in electrophoresis. Despite the fact that none of these deformations belongs to the functional space spanned by the B-spline deformation model, we obtain subpixel accuracy in both cases. The average warping index in all the simulations was below a tenth of a pixel.

Landmarks play a central role in registration of images with low information content, with large deformations, or when the information is unevenly distributed. In the presence of pointwise landmarks, one purpose of regularization is to provide a recipe to help determine the deformation in the area between them. Several authors have already pointed out that the regularization framework introduced in [29] is particularly useful in image registration [10], [23], [30]. Among the approaches using B-splines, the explicit use of landmarks was considered only in [12] and [55]. We have extended that work by introducing an initialization of the optimization process that takes into account the regularization constraints. This initialization is the optimal solution to the landmark regularized registration problem within our space of admissible deformations. We have given efficient formulæ for its computation in terms of the B-spline coefficients.

This regularization scheme offers new opportunities to incorporate a priori knowledge. For instance, we have proposed plausible deformation models for which the regularizing weights clearly depart from those that would emulate the more traditional Laplacian-based regularization. A priori knowledge is a key piece of information in the registration of images with a low information content.

The landmark-only registration problem becomes even more difficult when the correspondence between landmarks is unknown, as is the case with 2-D-gel spot matching. We showed in Table I that part of the problem comes from the necessity of using a high number of landmarks if high registration accuracy is to be achieved without data term (the information contained in the image itself). However, if the 2-D gel itself is used as indicated in [52], and as in our own experiments, then the number 


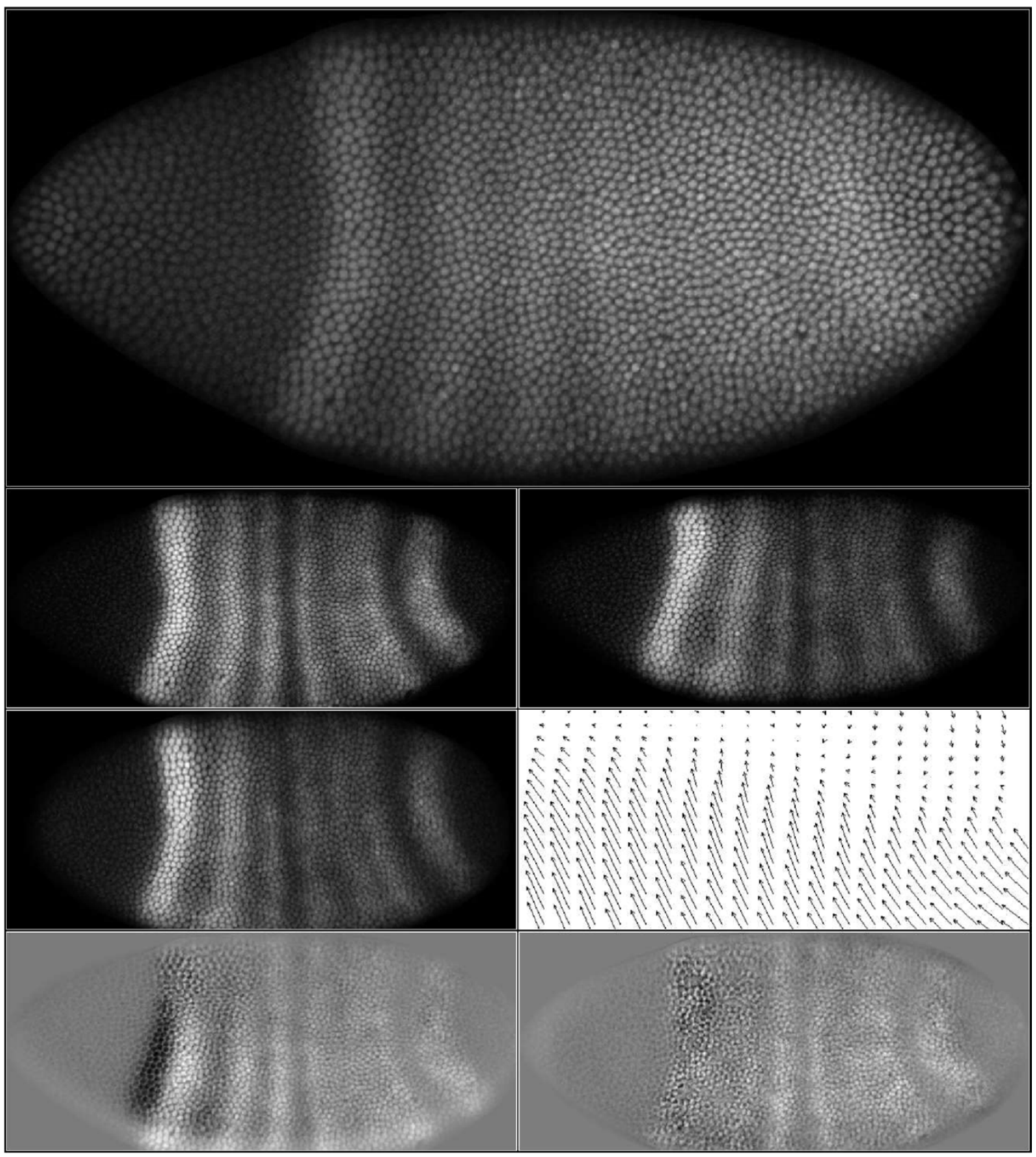

Fig. 9. From top to down, left to right: confocal scanning image of a sample embryo, target image, source image, registered image, recovered deformation field, difference image before registration, difference image after registration.

of landmarks can be strongly reduced. We also showed that if the image term is ignored, then our a priori knowledge about the nature of the deformation may account for as much as one half of the number of needed landmarks.

Contrary to most medical images, it often happens in the context of biological images that extended image regions bear no relevant information (e.g., gaps between spots in an electrophoretic gel). For these images, a registration method based on image-only contributions fails to achieve sufficient robustness, while too many landmarks are required for a landmark-only registration method to be serviceable. A combination of landmark terms and of other contributions that depend directly on the image data allows us to fill the gap between fully automatic and fully interactive registration. Generally speaking, the reg- istration algorithm is not very sensible to the choice of the free parameters $w_{i}, w_{\mu}, w_{d}$, and $w_{r}$ when enough information is supplied to the algorithm. For instance, some experiments were carried out to find the deformation field between two images when one of them was a $20^{\circ}$ rotated version of the other (results not shown). If no landmark was provided, the transformation was found only for very few combinations of the regularization weights. However, if a couple of landmarks was given or the rotation was smaller (in this case, the pixel values have enough information to drive the minimization), then the choice of the relative weights was not that consequential. To facilitate the exploration of these tradeoffs, we have developed a Java plugin for the platform-independent, public-domain software ImageJ which is particularly rich in tools for processing 
images, including those of electrophoretic gels. The plugin is freely available from our website http://bigwww.epfl.ch/. The computation time of the algorithm depends on the image size, the image content, the information provided by the landmarks and the regularization, but times between $45 \mathrm{~s}$ and $150 \mathrm{~s}$ should be expected for images of size $512 \times 512$ pixels on a Pentium IV $1.2 \mathrm{GHz}$.

We have also introduced the combined use of the optimization algorithm proposed by [34] with the BFGS Hessian estimator. This combination greatly enhances the performance of the optimizer while benefitting from its balance between quasi-Newton steps when the Hessian provides useful information for the minimization and steepest-descent steps when it does not.

The extension of this algorithm to three-dimensional (3-D) could use the same objective function as in (1) since all its terms are well-defined for 3-D deformation fields. The computation of the regularization term and its derivatives would be much more complicated, although thanks to the B-spline representation of the deformation field it would also result in a quadratic form. Therefore, the computation of the deformation field in the landmark-only registration case would still be valid as well as the optimizer for the full registration problem.

The application to experimental images of 2-D gels and of fly embryos showed its applicability to real biological problems. The evaluation of the registration results by experts in those respective fields favors our algorithm versus current standard approaches. Further collaborations are underway to produce biologically significant results in both applications.

\section{ACKNOWLEDGMENT}

The authors would like to thank Dr. R. Appel and Dr. D. Walther of the Swiss Institute of Bioinformatics at Geneva for letting them access their 2-D gel images and for their expert evaluation of the results. They would also like to thank Dr. M. Samsonova of the Department of Computational Biology at the Center for Advanced Studies of Saint Petersburg State Polytechnical University for providing the fly embryo images and for further evaluating the results.

\section{REFERENCES}

[1] B. Zitova and J. Flusser, "Image registration methods: A survey," Image Vis. Computing, vol. 21, no. 11, pp. 977-1000, Oct. 2003.

[2] L. G. Brown, "A survey of image registration techniques," ACM Computing Surveys, vol. 24, no. 4, pp. 325-376, Dec. 1992.

[3] K. R. Hess, W. Zhang, K. A. Baggerly, D. N. Stivers, and K. R. Coombes, "Microarrays: Handling the deluge of data and extracting reliable information," Trends Biotechnol., vol. 19, no. 11, pp. 463-468, Nov. 2001.

[4] E. Myasnikova, A. Samsonova, K. Kozlov, M. Samsonova, and J. Reinitz, "Registration of the expression patterns of drosophila: Segmentation genes by two independent methods," Bioinformatics, vol. 17, no. 1, pp. 3-12, Jan. 2001.

[5] X. Pennec and N. Ayache, "A geometric algorithm to find small but highly similar 3D substructures in proteins," Bioinformatics, vol. 14, no. 6, pp. 516-522, Jun. 1998.

[6] A. W. Dowsey, M. J. Dunn, and G.-Z. Yang, "The role of bioinformatics in two-dimensional gel electrophoresis," Proteomics, vol. 3, no. 8, pp. 1567-1596, Aug. 2003.

[7] T. Akutsu, K. Kanaya, A. Ohyama, and A. Fujiyama, "Point matching under nonuniform distortions," Discrete Appl. Math., vol. 127, no. 1, pp. 5-21, Apr. 2003.

[8] A. M. Bazen and S. H. Gerez, "Fingerprint matching by thin-plate spline modeling of elastic deformations," Pattern Recogn., vol. 36, no. 8, pp. 1859-1867, Aug. 2003.
[9] K. Rohr, H. S. Stiehl, R. Sprengel, T. M. Buzug, J. Weese, and M. H. Kuhn, "Landmark-based elastic registration using approximating thinplate splines," IEEE Trans. Med. Imag., vol. 20, no. 6, pp. 526-534, Jun. 2001.

[10] D. Suter and F. Chen, "Left ventricular motion reconstruction based on elastic vector splines," IEEE Trans. Med. Imag., vol. 19, no. 4, pp. 295-305, Apr. 2000.

[11] J. Pánek and J. Vohradský, "Point pattern matching in the analysis of two-dimensional gel electropherograms," Electrophoresis, vol. 20, no. 18, pp. 3483-3491, Dec. 1999.

[12] J. Kybic and M. Unser, "Fast parametric elastic image registration," IEEE Trans. Image Process., vol. 12, no. 11, pp. 1427-1442, Nov. 2003.

[13] D. Mattes, D. R. Haynor, H. Vesselle, T. K. Lewellen, and W. Eubank, "PET-CT image registration in the chest using free-form deformations," IEEE Trans. Med. Imag., vol. 22, no. 1, pp. 120-128, Jan. 2003.

[14] O. Musse, F. Heitz, and J.-P. Armspach, "Fast deformable matching of 3D images over multiscale nested subspaces. Application to atlas-based MRI segmentation," Pattern Recogn., vol. 36, no. 8, pp. 1881-1899, Aug. 2003.

[15] M. Unser, A. Aldroubi, and M. Eden, "Fast B-spline transforms for continuous image representation and interpolation," IEEE Trans. Pattern Anal. Mach. Intell., vol. 13, no. 3, pp. 277-285, Mar. 1991.

[16] R. Szeliski and H.-Y. Shum, "Motion estimation with quadtree splines," IEEE Trans. Pattern Anal. Mach. Intell., vol. 18, no. 12, pp. 1199-1210, Dec. 1996.

[17] M. Unser, "Splines: A perfect fit for signal and image processing," IEEE Signal Process. Magazine, vol. 16, no. 6, pp. 22-38, Nov. 1999.

[18] E. Meijering, W. Niessen, and M. Viergever, "Quantitative evaluation of convolution-based methods for medical image interpolation," Med. Image Anal., vol. 5, no. 2, pp. 111-126, Jun. 2001.

[19] P. Thévenaz, T. Blu, and M. Unser, "Interpolation revisited," IEEE Trans. Med. Imag., vol. 19, no. 7, pp. 739-758, Jul. 2000.

[20] R. Szeliski and J. Coughlan, "Spline-based image registration," International J. Comput. Vis., vol. 22, no. 3, pp. 199-218, Mar./Apr. 1997.

[21] G. E. Christensen, R. D. Rabbitt, and M. I. Miller, "Deformable templates using large deformation kinematics," IEEE Trans. Image Process., vol. 5, no. 10, pp. 1435-1447, Oct. 1996.

[22] G. E. Christensen, M. I. Miller, M. W. Vannier, and U. Grenander, "Individualizing neuroanatomical atlases using a massively parallel computer," Computer, vol. 29, no. 1, pp. 32-38, Jan. 1996.

[23] F. Chen and D. Suter, "Image coordinate transformation based on DIVCURL vector splines," in Proc. 14th Int. Conf. Pattern Recognition, (ICPR'98), vol. I, Brisbane, Australia, Aug. 16-20, 1998, pp. 518-520.

[24] T. Rohlfing, C. R. Maurer, D. A. Bluemke, and M. A. Jacobs, "Volumepreserving nonrigid registration of MR breast images using free-form deformation with an incompressibility constraint," IEEE Trans. Med. Imag., vol. 22, no. 6, pp. 730-741, Jun. 2003.

[25] J. Kybic, P. Thevénaz, and M. Unser, "Compensation of unidirectional geometric distortion in EPI using spline warping," in Proc. 1999 IEEE Int. Conf. Image Processing (ICIP'99), vol. II, Kobe, Japan, Oct. 25-28, 1999, pp. 168-172.

[26] S. Henn and K. Witsch, "A multigrid approach for minimizing a nonlinear functional for digital image matching," Computing, vol. 64, no. 4, pp. 339-348, Jun. 2000

[27] D. Rueckert, L. I. Sonoda, C. Hayes, D. L. G. Hill, M. O. Leach, and D. J. Hawkes, "Non-rigid registration using free-form deformations: Application to breast MR images," IEEE Trans. Med. Imag., vol. 18, no. 8, pp. 712-721, Aug. 1999.

[28] F. L. Bookstein, "Principal warps: Thin-plate splines and the decomposition of deformations," IEEE Trans. Pattern Anal. Mach. Intell., vol. 11, no. 6, pp. 567-585, Jun. 1989.

[29] L. Amodei and M. N. Benbourhim, "A vector spline approximation," $J$. Approximation Theory, vol. 67, no. 1, pp. 51-79, Jan. 1991.

[30] D. Suter, "Motion estimation and vector splines," in Proc. 1994 IEEE Conf. Computer Vision and Pattern Recognition (CVPR'94), Seattle, WA, Jun. 21-23, 1994, pp. 939-942.

[31] B. Fischer and J. Modersitzki, "Combination of automatic nonrigid and landmark based registration: The best of both worlds," in Med. Imag.: Image Process., M. Sonka and J. M. Fitzpatrick, Eds., 2003, vol. 5032, pp. 1037-1048.

[32] H. Johnson and G. Christensen, "Consistent landmark and intensity based image registration," IEEE Trans. Med. Imag., vol. 21, no. 5, pp. 450-461, May 2002.

[33] K. Rohr, P. Cathier, and S. Worz, "Elastic registration of electrophoresis images using intensity information and point landmarks," Pattern Recogn., vol. 37, no. 5, pp. 1035-1048, May 2004. 
[34] P. Thevénaz, U. E. Ruttimann, and M. Unser, “A pyramid approach to subpixel registration based on intensity," IEEE Trans. Image Process., vol. 7, no. 1, pp. 27-41, Jan. 1998.

[35] W. Press, S. A. Teukolsky, W. T. Vetterling, and B. P. Flannery, Eds., Numerical Recipes in $C$, 2nd ed. Cambridge, U.K.: Cambridge Univ. Press, 1992.

[36] F. Maes, A. Collignon, D. Vandermeulen, G. Marchal, and P. Suetens, "Multimodality image registration by maximization of mutual information," IEEE Trans. Med. Imag., vol. 16, no. 2, pp. 187-198, Apr. 1997.

[37] M. Unser, A. Aldroubi, and M. Eden, "The L2-polynomial spline pyramid," IEEE Trans. Pattern Anal. Mach. Intell., vol. 15, no. 4, pp. 364-379, Apr. 1993.

[38] C. O. S. Sorzano, L. G. de la Fraga, R. Clackdoyle, and J. M. Carazo, Normalizing projection images: A study of image normalizing procedures for single particle three-dimensional electron microscopy, in Ultramicroscopy, vol. 101, pp. 129-138, 2004.

[39] M. Unser, A. Aldroubi, and M. Eden, "B-spline signal processing: Part II-Efficient design and applications," IEEE Trans. Signal Process., vol. 41, no. 2, pp. 834-848, Feb. 1993.

[40] M. Unser and T. Blu, "Mathematical properties of the JPEG2000 wavelet filters," IEEE Trans. Image Process., vol. 12, no. 9, pp. 1080-1090, Sep. 2003.

[41] S. J. Colley, Vector Calculus, 2nd ed. Upper Saddle River, NJ: Prentice-Hall, 2001.

[42] J. E. Dennis and R. B. Schnabel, Numerical Methods for Unconstrained Optimization and Nonlinear Equations, 2nd ed. Philadelphia, PA: SIAM, 1996.

[43] L. Ma, Y. Q. Chen, and K. L. Moore, "Flexible camera calibration using a new analytical radial undistortion formula with application to mobile robot localization," presented at the 2003 IEEE Int. Symp. Intelligent Control (ISIC'03), Houston, TX, Oct. 5-8, 2003.

[44] "Mini Protean 3: Electrophoresis Instruction Manual," Biorad Laboratories, Hercules, CA, 2003.

[45] B. Raman, A. Cheung, and M. R. Marten, "Quantitative comparison and evaluation of two commercially available, two-dimensional electrophoresis image analysis software packages, Z3 and Melanie," Electrophoresis, vol. 23, no. 14, pp. 2194-2202, Nov. 2002.

[46] R. D. Appel, J. R. Vargas, P. M. Palagi, D. Walther, and D. F. Hochstrasser, "Melanie II-A third-generation software package for analysis of two-dimensional electrophoresis images: II. Algorithms," Electrophoresis, vol. 18, no. 15, pp. 2735-2748, Dec. 1997.

[47] K. Kriegel, I. Seefeldt, F. Hoffmann, C. Schultz, C. Wenk, V. RegitzZagrosek, H. Oswald, and E. Fleck, "An alternative approach to deal with geometric uncertainties in computer analysis of two-dimensional electrophoresis gels," Electrophoresis, vol. 21, no. 13, pp. 2637-2640, Jul. 2000.

[48] P. F. Lemkin, "Comparing two-dimensional electrophoretic gel images across the internet," Electrophoresis, vol. 18, pp. 461-470, 1997.

[49] J. Salmi, T. Aittokallio, J. Westerholm, M. Griese, A. Rosengren, T. A. Nyman, R. Lahesmaa, and O. Nevalainen, "Hierarchical grid transformation for image warping in the analysis of two-dimensional electrophoresis gels," Proteomics, vol. 2, no. 11, pp. 1504-1515, Nov. 2002.

[50] J. S. Gustafsson, A. Blomberg, and M. Rudemo, "Warping two-dimensional electrophoresis gel images to correct for geometric distortions of the spot pattern," Electrophoresis, vol. 23, pp. 1731-1744, Jun. 2002.

[51] Z. Smilansky, "Automatic registration for images of two-dimensional protein gels," Electrophoresis, vol. 22, no. 9, pp. 1616-1626, May 2001.

[52] S. Veeser, M. J. Dunn, and G.-Z. Yang, "Multiresolution image registration for two-dimensional gel eletrophoresis," Proteomics, vol. 1, no. 7, pp. 856-870, Jul. 2001

[53] S. Sternberg, "Biomedical image processing," Computer, vol. 16, no. 1, pp. 22-34, Jan. 1983.

[54] K. Kozlov, E. Myasnikova, A. Pisarev, M. Samsonova, and J. Reinitz, “A method for two-dimensional registration and construction of the two-dimensional atlas of gene expression patterns in situ," Silica Biol. (Special Issue: Dagstuhl Seminar "Functional Genomics"), vol. 2, no. 11, pp. 125-141, Feb. 2002.

[55] T. Hartkens, D. L. G. Hill, A. D. Castellano-Smith, D. J. Hawkes, C. R. Maurer, A. J. Martin, W. A. Hall, H. Liu, and C. L. Truwit, "Using points and surfaces to improve voxel-based nonrigid registration," in Lecture Notes in Computer Science, T. Dohi and R. Kikinis, Eds. Berlin, Germany, Sep. 2002, vol. 2489, Proceedings MICCAI, pp. 565-572.

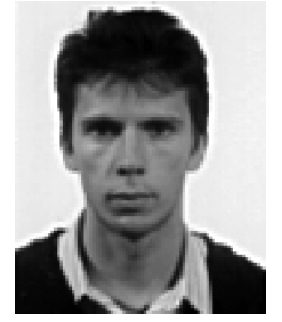

Carlos Óscar S. Sorzano was born in 1973 in Malaga, Spain. He receive the M.Sc. degree in electrical engineering and the B.Sc. degree in computer science from the University of Málaga, in 1997 and 2000 , respectively. He received the Ph.D. degree in 2002 from Univ. Politécnica de Madrid, with a thesis on the use of iterative reconstruction algorithms for single particles.

He joined the National Center of Biotechnology (CSIC, Madrid, Spain) in 1997, where he worked on electron microscopy of single particles. From 2003 to 2004, he worked as a Research Assistant in the Biomedical Imaging Group, Swiss Federal Institute of Technology Lausanne, Switzerland, where he developed image registration algorithms. He then joined the University of San Pablo CEU where he continues his research activity on electron microscopy and teaches at the Polytechnic School.

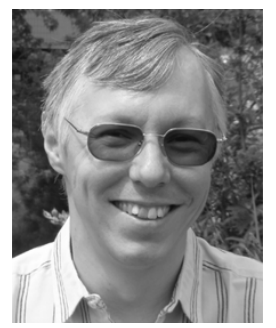

Philippe Thévenaz was born in 1962 in Lausanne, Switzerland. He graduated from the Lausanne Swiss Federal Institute of Technology (EPFL) in January 1986, with a diploma in microengineering. He then joined the Institute of Microtechnology (IMT) of the University of Neuchâtel, Switzerland, where he worked as Research Assistant and Scientist, first in the domain of image processing (optical flow), then in the domain of speech processing (speech coding and speaker recognition). He received the Ph.D. degree in June 1993, with a thesis on the use of the linear prediction residue for text-independent speaker recognition.

$\mathrm{He}$ then worked as a Visiting Fellow with the Biomedical Engineering and Instrumentation Program, National Institutes of Health (NIH), Bethesda, MD, where he developed research interests that include splines and multi-resolution signal representations, geometric image transformations, and biomedical image registration. Since November 1998, he is with the Swiss Federal Institute of Technology Lausanne as first assistant, where he extends his activities to research in interpolation and in volume rendering, and to mentoring of Ph.D. degree students.

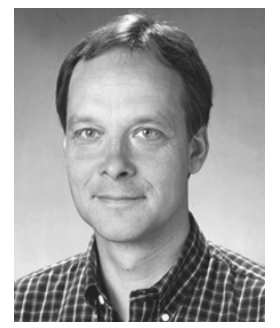

Michael Unser (M'89-SM'94-F'99) received the M.S. (summa cum laude) and Ph.D. degrees in electrical engineering in 1981 and 1984, respectively, from the Swiss Federal Institute of Technology in Lausanne (EPFL), Switzerland.

From 1985 to 1997, he was with the Biomedical Engineering and Instrumentation Program, National Institutes of Health, Bethesda, MD, where he was heading the Image Processing Group. He is now Professor and Director of the Biomedical Imaging Group at the EPFL. His main research area is biomedical image processing. He has a strong interest in sampling theories, multiresolution algorithms, wavelets, and the use of splines for image processing. He is the author of over 100 published journal papers in these areas.

Dr. Unser is the Associate Editor-in-Chief of the IEEE TRANSACTIONS on Medical IMAgING and the Editor-in-Chief of the Wavelet Digest, the electronic newsletter of the wavelet community. He has acted as associate editor or member of the editorial board for eight more international journals, including the IEEE SignAL PROCESSING Magazine, the IEEE TRANSACTIONS ON IMAGE PROCESSING (1992-1995), and the IEEE SignAl Processing LETTERS (1994-1998). He serves as regular chair for SPIE's conference on Wavelets, which has been held annually since 1993 . He was general co-chair for the first IEEE International Symposium on Biomedical Imaging (ISBI'2002), which was held in Washington DC, July 7-10, 2002. He received three best paper awards from the IEEE Signal Processing Society: the 1995 and 2003 Best Paper Awards, and the 2000 Magazine Award. In January 1999, he was elected Fellow of the IEEE with the citation: "for contributions to the theory and practice of splines in signal processing." 